\title{
The coupled-cluster method applied to the $X X Z$ model using a planar model state
}

\author{
R F Bishop, D J J Farnell and J B Parkinson \\ Department of Physics, University of Manchester Institute of Science and Technology (UMIST), \\ PO Box 88, Manchester M60 1QD, UK
}

Received 2 August 1996

\begin{abstract}
The coupled-cluster method (CCM) is applied to the spin- $\frac{1}{2} X X Z$ model for the 1D chain and the 2D square lattice in the region $-1<\Delta<1$. A CCM model state is chosen in which all spins lie in the $x y$ plane with nearest-neighbour spins anti-parallel. Very good agreement with the exactly known ground-state energies in $1 \mathrm{D}$ is obtained, and good agreement with Monte Carlo results is obtained for the 2D case. The sublattice magnetization is obtained, and evidence of phase transition points in both $1 \mathrm{D}$ and 2D is presented. The critical behaviour of the 1D ground-state energy near the ferromagnetic phase transition point is investigated.
\end{abstract}

\section{Introduction}

The coupled-cluster method (CCM) [1-9] is a formulation of quantum many-body theory which has been applied to many different physical problems with great success. It has recently been applied to such quantum spin systems as the Heisenberg model [10], the $X X Z$ model $[11,12]$, the $J_{1}-J_{2}$ model $[13,14]$, and the Heisenberg-biquadratic model [15]. The higher LSUB $n$ approximations applied to the $X X Z$ model [12] have in particular provided very good estimates of the ground-state energies and the positions of the phase transition points of this system. The research presented here extends the previous treatment of the $X X Z$ model via the CCM by describing a more realistic CCM treatment of a phase of the system in which the spins are believed to lie in the $x y$ plane.

The Hamiltonian that we shall consider is the spin- $\frac{1}{2} X X Z$ Hamiltonian for the onedimensional (1D) chain or the two-dimensional (2D) square lattice with periodic boundary conditions,

$$
H=\frac{1}{2} \sum_{i=1}^{N} \sum_{\rho}\left[s_{i}^{x} s_{i+\rho}^{x}+s_{i}^{y} s_{i+\rho}^{y}+\Delta s_{i}^{z} s_{i+\rho}^{z}\right] .
$$

Note that the index $i$ in equation (1) runs over all $N$ lattice sites, and $\rho$ runs over all $z$ nearest neighbours to a given site for both the $1 \mathrm{D}$ chain $(z=2)$ and the 2D square lattice $(z=4)$.

For the 2D case, the $X X Z$ model has no exact solution although many approximate analytical [16-18] and numerical calculations [19-21] have been performed. At $\Delta=1$, Runge [19] has performed the most accurate Monte Carlo simulation with a value for the ground-state energy of $-0.66934(4)$, and a value for the sublattice magnetization which is $61.5 \% \pm 0.5 \%$ of the classical value. In comparison, linear spin-wave theory (LSWT) [16] gives values of -0.658 for the ground-state energy, and yields a value which is $60.6 \%$ of the classical value for the sublattice magnetization. Away from the isotropic point, a Monte 
Carlo study of the 2D anisotropic Heisenberg model has been undertaken by Barnes et al [21]. They observed that the staggered magnetization appears to become zero for $\Delta \leqslant 1$ (at $\Delta=-1$ there is a first-order phase transition to the ferromagnetic phase), and they also conclude that the critical point is probably very near to $\Delta=1$. In contrast to this Monte Carlo calculation, Kubo and Kishi [18] have used sum rules to investigate the ground state of this system. They state that the ground state possesses an off-diagonal long-range order (LRO) akin to that of the $X Y$-like state at small anisotropy, $0.0<\Delta<0.13$. Also, for $\Delta>1.78$ they observe that the system demonstrates non-zero Ising-like LRO.

In contrast to the $2 \mathrm{D}$ case, the $X X Z$ chain has been solved exactly via the well known Bethe ansatz [22-24]. The ground state of the 1D $X X Z$ model displays non-zero Néel-type LRO for $\Delta>1$, and at the isotropic point $\Delta=1$ there is an infinite-order phase transition from the Néel-type regime to a critical regime in which no Néel-type LRO is present. The critical regime in $1 \mathrm{D}$ extends over the range $-1<\Delta<1$, and at $\Delta=-1$ there is a first-order phase transition to the ferromagnetic ground state.

\section{The ground-state energy}

We begin the CCM treatment of this spin system by choosing a suitable model state $|\Phi\rangle$ such that all other possible spin configurations may be obtained by the application of linear combinations of products of spin-raising operators to this state. For the $X X Z$ model in the region $-1<\Delta<1$ we use the classical Néel state but with spins constrained to lie along the $x$-axis. In $1 \mathrm{D}$, the model state is therefore illustrated by

$$
|\Phi\rangle=|\ldots \leftarrow \rightarrow \leftarrow \rightarrow \leftarrow \rightarrow \leftarrow \rightarrow \leftarrow \rightarrow \ldots\rangle .
$$

In order to treat the spins on each sublattice equivalently we now rotate the local axes of these spins on the separate sublattices such that they appear to be lying in the negative $z$-direction. This is achieved by rotating the axes of the left-pointing spins (i.e., those pointing along the negative $x$-direction) by $90^{\circ}$ about the $y$-axis, and by rotating the axes of the right-pointing spins (i.e., those pointing along the positive $x$-direction) by $270^{\circ}$ about the $y$-axis. The transformation of the local axes of the left-pointing spins is therefore

$$
s^{x} \rightarrow s^{z} \quad s^{y} \rightarrow s^{y} \quad s^{z} \rightarrow-s^{x}
$$

and the transformation of the local axes of the right-pointing spins is

$$
s^{x} \rightarrow-s^{z} \quad s^{y} \rightarrow s^{y} \quad s^{z} \rightarrow s^{x} .
$$

Hence our model state now appears mathematically to have all spins pointing downwards.

Furthermore, it is useful to use the Pauli operators which are related to the spin operators by the relationship $\sigma^{\alpha}=2 s^{\alpha}(\alpha=x, y, z)$. The usual commutation relations for the Pauli spin operators still remain valid regardless of sublattice, namely

$$
\begin{aligned}
& {\left[\sigma_{l}^{z}, \sigma_{l^{\prime}}^{ \pm}\right]= \pm 2 \sigma_{l}^{ \pm} \delta_{l, l^{\prime}}} \\
& {\left[\sigma_{l}^{+}, \sigma_{l^{\prime}}^{-}\right]=\sigma_{l}^{z} \delta_{l, l^{\prime}} .}
\end{aligned}
$$

Note that we define the spin-raising and spin-lowering operators as $\sigma_{l}^{ \pm} \equiv \frac{1}{2}\left(\sigma_{l}^{x} \pm \mathrm{i} \sigma_{l}^{y}\right)$. The application of these transformations will affect our Hamiltonian, which may now be re-written in terms of the Pauli operators, using the new rotated local spin axes, as

$$
H=-\frac{1}{8} \sum_{i=1}^{N} \sum_{\rho}\left[(\Delta+1)\left(\sigma_{i}^{+}{\sigma_{i+\rho}^{+}}^{+}+\sigma_{i}^{-}{\sigma_{i+\rho}^{-}}^{-}\right)+(\Delta-1)\left(\sigma_{i}^{+}{\sigma_{i+\rho}^{-}}^{-}+\sigma_{i}^{-}{\sigma_{i+\rho}^{+}}^{+}\right)+\sigma_{i}^{z} \sigma_{i+\rho}^{z}\right] \text {. }
$$


In order to obtain quantities such as the ground-state energy we must define the ket ground-state wavefunction. This state is constructed using the CCM ansatz, given by

$$
|\Psi\rangle=\mathrm{e}^{S}|\Phi\rangle \text {. }
$$

$S$ is called the CCM ket-state correlation operator, and may be written as

$$
S=\sum_{I} S^{I} C_{I}^{\dagger}
$$

where $C_{I}^{\dagger}$ are a complete set of creation operators with respect to our model state. Each $C_{I}^{\dagger}$ consists of a product of spin-raising operators on a number of sites, and the $S^{I}$ are $c$-number ket-state correlation coefficients.

Once the ket state has been defined it is possible to obtain an expression for the groundstate energy by utilizing the Schrödinger equation, $E_{g}|\Psi\rangle=H|\Psi\rangle$, such that

$$
E_{g}=\left\langle\Phi\left|\mathrm{e}^{-S} H \mathrm{e}^{S}\right| \Phi\right\rangle .
$$

Equation (10) involves the similarity transform of $H$, which is a key feature of a CCM calculation, and it is often useful to expand this expression in terms of a series of nested commutators, given by

$$
\mathrm{e}^{-S} H \mathrm{e}^{S}=H+[H, S]+\frac{1}{2 !}[[H, S], S]+\cdots .
$$

A notable feature of the CCM approach is that, because the Hamiltonian of equation (7) contains only finite sums of products of spin operators, this series must terminate after a finite number of terms. Another natural consequence of the series expansion is that every raising operator in $S$ is linked to a Pauli spin operator in $H$. The expression for the groundstate energy in equation (10) therefore obeys the linked-cluster theorem, and so the value for the ground-state energy per spin is automatically size-extensive, and we may safely take the infinite lattice limit $(N \rightarrow \infty)$ from the outset.

It is often necessary to make an approximation in our $S$ operator for a practical application of the CCM, and in this paper we use two approximation schemes. However, before the details of these schemes are discussed, we shall consider how the Hamiltonian of equation (7) influences our choice of which correlations to include in $S$. $H$ contains products of even numbers of spin-raising and spin-lowering operators, and also a single term formed from $\sigma^{z}$ operators. Repeated application of $H$ to the model state eventually yields the ground state (assuming that the model state is not orthogonal to it). Hence we may conclude that the ground state of the system must contain an even number of spin up and spin down states with respect to $|\Phi\rangle$, and we also may simplify our problem by including only terms with an even numbers of Pauli spin-raising operators in $S$.

The first approximation scheme that we shall consider is called the LSUBn approximation scheme. The LSUBn scheme contains all possible (connected and disconnected) terms in $S$ which are contained within a 'locale' of size $n$. In both 1D and $2 \mathrm{D}$, we use all possible connected configurations of $n$ spins to define this locale; in $1 \mathrm{D}$ we may see that this locale is simply a chain of length $n$. Disconnected and connected configurations of less than $n$ spins are then generated by successively removing sites from the original connected configurations of $n$ spins, thus covering all possibilities. The lowest-order LSUB $n$ approximation scheme is the LSUB2 approximation in which only a single nearestneighbour, two-body term is retained in equation (9). The LSUB2 correlation operator in both $1 \mathrm{D}$ and $2 \mathrm{D}$ may be compactly written as

$$
S=\frac{1}{2} b_{1} \sum_{i=1}^{N} \sum_{\rho} \sigma_{i}^{+} \sigma_{i+\rho}^{+} .
$$


The index $i$ once again runs over all lattice points, and $\rho$ indicates nearest-neighbour lattice vectors. The ground-state energy of equation (10) may now be derived explicitly in terms of $b_{1}$ by evaluating the similarity transform in terms of the series of nested commutators of equation (11). Thus for LSUB2 we obtain

$$
\frac{E_{g}}{N}=-\frac{z}{8}\left[b_{1}(\Delta+1)+1\right]
$$

where $z$ is the coordination number. In fact this expression for the ground-state energy is correct for any choice of $S$ in equation (10), including the exact untruncated expression. Our choice of $S$ will affect the value of $b_{1}$ however.

We now wish to obtain a numerical value for the $b_{1}$ coefficient. This is achieved by evaluating the CCM ket-state equations, given by

$$
\left\langle\Phi\left|C_{I} \mathrm{e}^{-S} H \mathrm{e}^{S}\right| \Phi\right\rangle=0
$$

where the $C_{I}$ are the Hermitian conjugates of the $C_{I}^{\dagger}$ retained in $S$ and are formed from Pauli spin-lowering operators. At the LSUB2 level of approximation we have a single term in $S$, and so we obtain a single ket-state equation by substituting $\sigma_{i}^{-} \sigma_{i+\rho}^{-}$for $C_{I}$ in equation (14). Hence in 1D we obtain

$$
(\Delta+1)\left(3 b_{1}^{2}-1\right)+4 b_{1}=0
$$

and in $2 \mathrm{D}$ we obtain

$$
(\Delta+1)\left(5 b_{1}^{2}-1\right)+12 b_{1}=0 .
$$

The ground-state energies obtained at this level of approximation are illustrated in figures 1 and 2 , and in tables 1 and 2 .

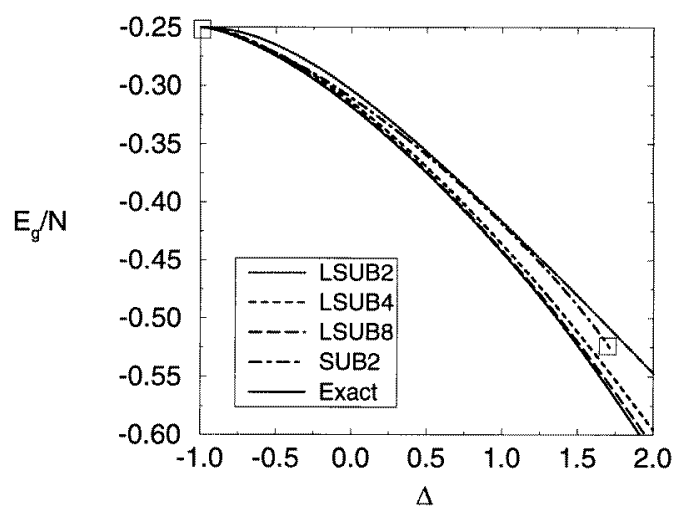

Figure 1. Results for the 1D CCM ground-state energy compared to the exact results. SUB2 critical points are indicated by the boxes.

The next level of approximation is the LSUB4 scheme, which in 1D contains four separate configurations. The 1D LSUB4 ket-state correlation operator is given by

$S=b_{1} \sum_{i=1}^{N} \sigma_{i}^{+} \sigma_{i+1}^{+}+b_{2} \sum_{i=1}^{N} \sigma_{i}^{+} \sigma_{i+2}^{+}+b_{3} \sum_{i=1}^{N} \sigma_{i}^{+} \sigma_{i+3}^{+}+g_{4} \sum_{i=1}^{N} \sigma_{i}^{+} \sigma_{i+1}^{+} \sigma_{i+2}^{+} \sigma_{i+3}^{+}$.

Evaluation of (14) for the LSUB4 approximation scheme follows in the same manner as for the LSUB2 approximation. Hence for the 1D LSUB4 scheme we obtain four coupled 


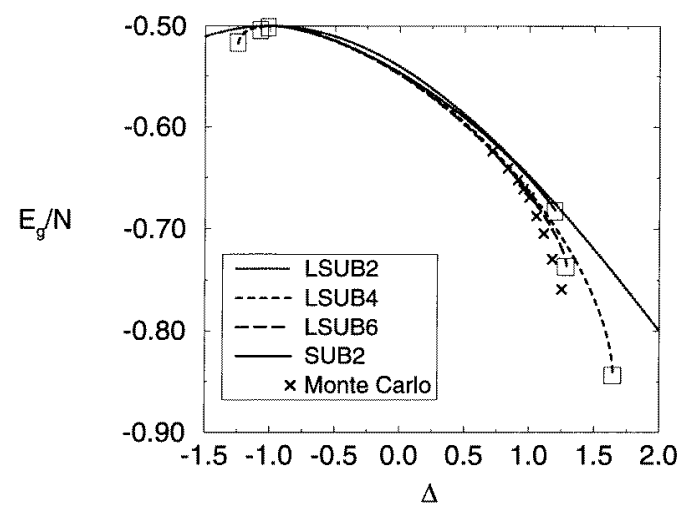

Figure 2. Results for the 2D CCM ground-state energy compared to the Monte Carlo results of [21]. SUB2 and LSUB $n$ critical points are indicated by the boxes.

Table 1. Results for the 1D CCM ground-state energy for the calculations based on the planar model state, compared to the exact results. CCM results based on the Neel model state with spins lying along the $z$-axis are also shown in parentheses.

\begin{tabular}{clllllll}
\hline$\Delta$ & LSUB2 & SUB2 & LSUB4 & LSUB6 & LSUB8 & LSUB10 & Exact \\
\hline-1.0 & -0.25 & -0.25 & -0.25 & -0.25 & -0.25 & -0.25 & -0.25 \\
& $(-0.25)$ & $(-)$ & $(-0.3211)$ & $(-0.4178)$ & $(-0.3074)$ & $(-0.4082)$ & \\
-0.5 & -0.2650 & -0.2720 & -0.2718 & -0.2733 & -0.2738 & -0.2741 & -0.2745 \\
& $(-0.2588)$ & $(-)$ & $(-0.2931)$ & $(-0.2797)$ & $(-0.2827)$ & $(-0.2763)$ & \\
0.0 & -0.3038 & -0.3104 & -0.3141 & -0.3164 & -0.3171 & -0.3175 & -0.3183 \\
& $(-0.2887)$ & $(-)$ & $(-0.3193)$ & $(-0.3198)$ & $(-0.3196)$ & $(-0.3194)$ & \\
0.5 & -0.3566 & -0.3596 & -0.3698 & -0.3725 & -0.3735 & -0.3740 & -0.3750 \\
& $(-0.3421)$ & $(-0.3506)$ & $(-0.3692)$ & $(-0.3730)$ & $(-0.3741)$ & $(-0.3745)$ & \\
1.0 & -0.4167 & -0.4186 & -0.4363 & -0.4400 & -0.4414 & -0.4420 & -0.4432 \\
& $(-0.4167)$ & $(-0.4186)$ & $(-0.4363)$ & $(-0.4400)$ & $(-0.4414)$ & $(-0.4420)$ & \\
1.5 & -0.4808 & -0.4893 & -0.5119 & -0.5180 & -0.5201 & -0.5212 & -0.5234 \\
& $(-0.5069)$ & $(-0.5075)$ & $(-0.5195)$ & $(-0.5218)$ & $(-0.5226)$ & $(-0.5230)$ & \multirow{2}{*}{2.0172} \\
& -0.5473 & - & -0.5960 & -0.6060 & -0.6100 & -0.6121 & -0.6172 \\
& $(-0.6076)$ & $(-0.6078)$ & $(-0.6155)$ & $(-0.6167)$ & $(-0.6170)$ & $(-0.6171)$ & \\
\hline
\end{tabular}

nonlinear equations given by

$$
\begin{aligned}
& (\Delta+1)\left(2 g_{4}-3 b_{1}^{2}+2 b_{2}^{2}+2 b_{1} b_{3}+2 b_{3}^{2}+1\right)+2 b_{2}(\Delta-1)-4 b_{1}=0 \\
& (\Delta+1)\left(2 b_{2} b_{3}-4 b_{1} b_{2}\right)+2(\Delta-1)\left(b_{1}+b_{3}\right)-8 b_{2}=0 \\
& (\Delta+1)\left(g_{4}-4 b_{1} b_{3}+b_{1}^{2}+b_{2}^{2}\right)+2(\Delta-1) b_{2}-8 b_{3}=0 \\
& (\Delta+1)\left(8 g_{4} b_{1}+2 g_{4} b_{3}+4 b_{2}^{2} b_{3}+4 b_{1} b_{3}^{2}\right)+(\Delta-1)\left(8 b_{1} b_{2}+4 b_{2} b_{3}\right)+4 g_{4}-8 b_{1} b_{3} \\
& \quad-4 b_{1}^{2}-12 b_{2}^{2}=0 .
\end{aligned}
$$

These nonlinear equations are easily solved numerically by tracking an exact solution to these equations at the point $\Delta=-1$; at this point we know that all of the ket-state correlation coefficients must be zero because our model state is a ground eigenstate of equation (7). In 2D, the LSUB4 approximation contains ten terms in $S$, and the results are shown in figure 2 and table 2. 
Table 2. Results for the 2D CCM ground-state energy for the calculations based on the planar model state, compared to the Monte Carlo results of [21].

\begin{tabular}{llllll}
\hline$\Delta$ & LSUB2 & SUB2 & LSUB4 & LSUB6 & Monte Carlo \\
\hline-1.0 & -0.5 & -0.5 & -0.5 & -0.5 & - \\
-0.5 & -0.5103 & -0.5149 & -0.5145 & -0.5151 & - \\
0.0 & -0.5403 & -0.5463 & -0.5473 & -0.5483 & - \\
0.5 & -0.5874 & -0.5909 & -0.5959 & -0.5975 & - \\
0.7143 & -0.6120 & -0.6144 & -0.6222 & -0.6242 & -0.624 \\
0.8333 & -0.6267 & -0.6288 & -0.6385 & -0.6408 & -0.641 \\
0.9091 & -0.6364 & -0.6385 & -0.6496 & -0.6523 & -0.652 \\
0.9524 & -0.6420 & -0.6443 & -0.6562 & -0.6591 & -0.661 \\
1.0 & -0.6483 & -0.6509 & -0.6637 & -0.6670 & -0.669 \\
\hline
\end{tabular}

The expectation energy of our model state over the region $-1<\Delta<1$ in $1 \mathrm{D}$ is -0.25 and in $2 \mathrm{D}$ it is -0.5 . From tables 1 and 2 we conclude that even at these simple levels of approximation the CCM calculations provide a much improved estimate of the groundstate energy with respect to the expectation energy of our model state. These energies are compared to the exact result in 1D, and the Monte Carlo results in 2D.

Higher-order LSUB $n$ calculations for both of these systems have also been successfully attempted with our model state. However, these calculations involve much tedious algebra, and so have been implemented computationally $\dagger$ up to the LSUB6 level of approximation in 2D and up to the LSUB10 level of approximation in 1D. The numbers of distinct multispin configurations retained in the correlation operator $S$ are, respectively, 131 for the 2D LSUB6 case and 151 for the 1D LSUB10 case. The ground-state energies provided by these LSUB $n$ calculations provide very good correspondence with Monte Carlo results in 2D and exact results in 1D. This is illustrated in figures 1 and 2, and in tables 1 and 2. In fact, the LSUB10 ground-state energies in 1D provide results which are indistinguishable by eye from the exact ground-state energy (hence LSUB10 is not included in figure 1). Table 1 also shows ground-state energies for the $1 \mathrm{D} X X Z$ model based on the Néel model state with spins constrained to lie parallel to the $z$-axis. We may see from table 1 that our CCM ground-state results based on the planar model state provide excellent estimates compared to exact results in the region $-1<\Delta<1$, and that they converge smoothly with increasing LSUB $n$ approximation level. This is in contrast to the corresponding CCM results based on the $z$-axis aligned Néel model state which do not provide good estimates and do not uniformly converge over all of the region $-1<\Delta<1$; though we may note that there is a crossover region near to the antiferromagnetic phase boundary at $\Delta=1$ over which CCM calculations for both model states perform well. We note that in the region $\Delta>1$ the CCM calculations based on the $z$-axis aligned Néel model state provide better results, again with an area of crossover near $\Delta=1$. In 2D, no equivalent comparison of the calculations for the ground-state energy via the two model states in the region $-1<\Delta<1$ can be made because the LSUB $n$ results based on the $z$-axis aligned Néel model state do not extend very far into this region. Analogously, the results for the planar model state do not extend very far into the region $\Delta>1$, as is discussed in the following paragraph. We therefore conclude that our new model state provides a much better starting point for the CCM calculations in the region $-1<\Delta<1$ than the previous Néel model state. 
A useful measure for detecting phase transitions within the LSUBn scheme is the socalled anisotropy susceptibility $\chi_{a}$ given by

$$
\chi_{a}=\frac{1}{N} \frac{\mathrm{d}^{2} E_{g}}{\mathrm{~d} \Delta^{2}} .
$$

In 1D, we find that at the LSUB10 level of approximation there is a divergence in $\chi_{a}$ which occurs at $\Delta=-1.002$. For lower approximations, we find that there are only strong peaks which grow in magnitude as the LSUB $n$ approximation level is increased. These peaks also tend to become closer to the true ferromagnetic phase transition point at $\Delta=-1$ as the LSUBn approximation level is increased. The reason why there is no divergence for orders lower than LSUB10 is because the longer-range correlations with respect to our model state become very important near $\Delta=-1$. LSUB10 is therefore the smallest local LSUB $n$ approximation which is powerful enough to cause a divergence in $\chi_{a}$. We find no evidence of such a divergence or any peaks near $\Delta=1$ which might correspond to the antiferromagnetic phase transition point. In fact this is not too surprising as there is an infinite-order phase transition at this point, and, as we shall show, it is only when longer-range, SUB2 correlations are included in $S$ that it is detected at all.

In contrast to this, however, in $2 \mathrm{D}$ we find that $\chi_{a}$ diverges at both a negative and a positive value of $\Delta$, corresponding to the ferromagnetic and antiferromagnetic phase transitions respectively, for approximation levels greater than LSUB2. These results are illustrated in table 3. Table 3 also shows LSUB $n$ critical points for the corresponding CCM calculations based on the Néel model state with spins lying along the $z$-axis, and these critical points again correspond to the antiferromagnetic phase transition. As we expect, the position of the negative-valued critical point for the planar model state becomes closer to the true ferromagnetic phase transition point of this system, which is at $\Delta=-1.0$, as the approximation level is increased. The positive-valued critical points, based on both model states, appear to converge with increasing approximation level and always bound the point $\Delta=1$, at which the true antiferromagnetic phase transition is believed to lie.

Table 3. Results for the critical points of the 2D CCM LSUB4, LSUB6 and SUB2 approximations. $\Delta_{1}^{P}$ and $\Delta_{2}^{P}$ correspond to the ferromagnetic and antiferromagnetic phase transitions respectively for the calculations based on the planar model state. $\Delta^{N}$ corresponds to the antiferromagnetic phase transition for the calculations based on the Néel model state with spins lying along the $z$-axis of $[11,12]$.

\begin{tabular}{llll}
\hline & $\Delta_{1}^{P}$ & $\Delta_{2}^{P}$ & $\Delta^{N}$ \\
\hline LSUB4 & -1.249 & 1.648 & 0.577 \\
LSUB6 & -1.083 & 1.286 & 0.763 \\
SUB2 & -1.0 & 1.204 & 0.799 \\
\hline
\end{tabular}

We now direct our attention to another approximation called the SUB2 approximation scheme which retains all possible two-body correlations in $S$. The SUB2 ket-state correlation operator is given by

$$
S=\frac{1}{2} \sum_{i=1}^{N} \sum_{r} b_{r} \sigma_{i}^{+} \sigma_{i+r}^{+} .
$$


Note that $i$ runs over all lattice points and $r$ runs over all distinct lattice vectors apart from $r=0$. We now apply equation (14) to obtain the full SUB2 equations which are given by

$$
\begin{gathered}
\sum_{\rho}\left(1-\delta_{r, 0}\right)\left[(\Delta+1) \sum_{s} b_{s} b_{s+r+\rho}-4(\Delta+1) b_{1} b_{r}+2(\Delta-1) b_{r+\rho}-4 b_{r}\right. \\
\left.+\left\{(\Delta+1)\left(2 b_{1}^{2}+1\right)+4 b_{1}\right\} \delta_{r, \rho}\right]=0 .
\end{gathered}
$$

The solutions of these equations are obtained via Fourier transform methods $\dagger$. The solution for $b_{1}$ is found to be given by

$$
b_{1}=\frac{1}{(2 \pi)^{d}} \int \mathrm{d}^{d} q\left[\frac{-b-\sqrt{b^{2}-4 a c}}{2 a}\right] \gamma(q)
$$

where $d$ denotes the dimensionality. Note that in $1 \mathrm{D} \gamma(q)=\cos (q)$ and in $2 \mathrm{D}$ $\gamma(q)=\frac{1}{2}\left(\cos \left(q_{x}\right)+\cos \left(q_{y}\right)\right)$. Furthermore, the expressions within the integrand, $a, b$ and $c$, are given by

$$
\begin{aligned}
& a=(\Delta+1) \gamma(q) \\
& b=-4(\Delta+1) b_{1}+2(\Delta-1) \gamma(q)-4 \\
& c=\left[(\Delta+1)\left(2 b_{1}^{2}+1\right)+4 b_{1}\right] \gamma(q)+2(1-\Delta) b_{1}-(\Delta+1) X_{1} .
\end{aligned}
$$

The function $X_{1}$ may also be defined in terms of an integral, namely

$$
X_{1}=\sum_{s} b_{s} b_{s+\rho}=\frac{1}{(2 \pi)^{d}} \int \mathrm{d}^{d} q\left[\frac{-b-\sqrt{b^{2}-4 a c}}{2 a}\right]^{2} \gamma(q) .
$$

Equations (22) and (24) can now be solved numerically as self-consistency relations in terms of $b_{1}$ and $X_{1}$. The SUB2 ground-state energies for the 1D and 2D cases are presented in tables 1 and 2 respectively, and again, the SUB2 energy provides a much improved estimate of the true ground-state energy compared with the expectation energy of the model state.

In general, the ground-state energies for the SUB2 approximation are higher than those energies for the high-order LSUB $n$ approximations. However, as one approaches the ferromagnetic boundary the SUB2 results begin to lie lower than the LSUB $n$ energies. In 1D, we find that SUB2 becomes more accurate than LSUBn approximations in comparison with the exact solution. We may investigate the critical behaviour of the CCM ground-state energies as the ferromagnetic phase transition at $\Delta=-1$ is approached, and compare to exact results. Assuming that near $\Delta=-1$ in $1 \mathrm{D}$ we have

$$
\frac{E_{g}}{N} \rightarrow-\frac{1}{4}-A(\Delta+1)^{\alpha}
$$

then plotting $\log \left|\left(E_{g} / N+0.25\right)\right|$ against $\log (\Delta+1)$ near this point gives us the critical index $\alpha$. This is illustrated in figure 3. We may see that the exact results and SUB2 results are indistinguishable to the eye, and both give a value of $\alpha=1.50 \pm 0.01$. In contrast, the LSUB $n$ series all give values of $\alpha=2.00 \pm 0.01$, and so we conclude that the SUB2 approximation captures the essential correlations necessary to the ground-state energy near the ferromagnetic phase transition point in 1D. We discuss the LSUB $n$ results further in section 4.

$\dagger$ A full explanation of the Fourier transform solution of the $X X Z$ model for the full SUB2 equations based on the corresponding $z$-axis aligned Néel state is given in [11]. 


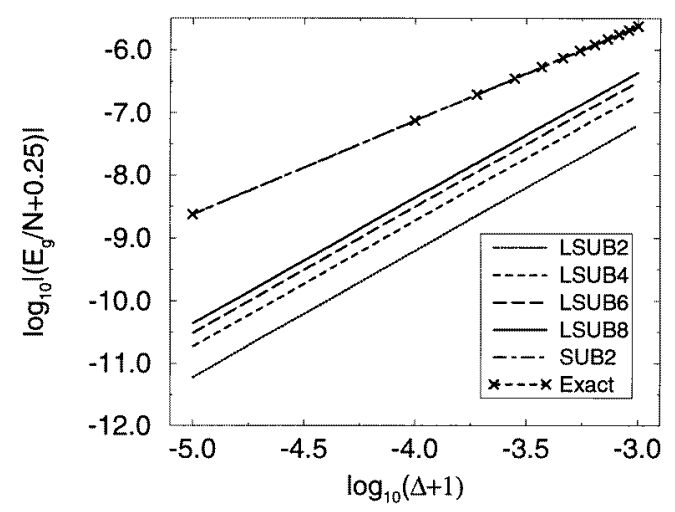

Figure 3. Results illustrating the critical behaviour of the 1D ground-state energy near the ferromagnetic phase transition point.

Another consequence of the full SUB2 calculation is that the discriminant of the square root in equation (22) becomes negative at certain values of $\Delta$, and these terminating or critical points are often taken as signatures of a phase transition point of the real system. In 1D, these points are found to be at $\Delta=-1.0$ and $\Delta=1.7126$. The lower point indicates the position of the ferromagnetic transition precisely, although the upper critical point is well away from the actual phase transition point at $\Delta=1$. However, the SUB2 scheme is the lowest order of approximation which contains infinitely long-range correlations and so perhaps this is not surprising-especially when we remember that the LSUB $n$ scheme did not even detect the antiferromagnetic phase transition in 1D. In 2D, we find that the SUB2 critical points are at $\Delta=-1.0$ and $\Delta=1.204$, as given in table 3 . As we might expect, this time the upper critical point compares more favourably to the position of the antiferromagnetic phase transition point, which in $2 \mathrm{D}$ is also believed to be near $\Delta=1.0$.

\section{The sublattice magnetization}

We shall now consider the bra state, which is parametrized via the normal coupled-cluster method (NCCM) $[6,8]$ by

$$
\langle\tilde{\Psi}|=\langle\Phi| \tilde{S} \mathrm{e}^{-S}
$$

where the bra-state correlation operator $\tilde{S}$ is given by

$$
\tilde{S}=1+\sum_{I} \tilde{S}^{I} C_{I} .
$$

The operators $C_{I}^{\dagger}$ are the same creation operators as in our $S$ operator, described by equation (9). The $C_{I}$ are the Hermitian conjugates of $C_{I}^{\dagger}$ and are thus formed purely from spin-lowering operators. The coefficient $\tilde{S}^{I}$ is the bra-state correlation coefficient associated with configuration $I$. Note that $\tilde{S}$ must be of the same order of approximation as the ket-state correlation operator $S$ so that the Hellmann-Feynman [25] theorem is satisfied. In analogy with equation (12), the bra-state correlation operator at the LSUB2 level of approximation is therefore given by

$$
\tilde{S}=1+\frac{1}{2} \tilde{b}_{1} \sum_{i=1}^{N} \sum_{\rho} \sigma_{i}^{-} \sigma_{i+\rho}^{-} .
$$


The bra-state equations may now be determined by using

$$
\left\langle\Phi\left|\tilde{S} \mathrm{e}^{-S}\left[H, C_{I}^{\dagger}\right] \mathrm{e}^{S}\right| \Phi\right\rangle=0
$$

which for LSUB2 in 1D yields

$$
(\Delta+1)\left(1-6 \tilde{b}_{1} b_{1}\right)-4 \tilde{b}_{1}=0 .
$$

The bra-state equations for higher-order LSUB $n$ approximations have also been calculated. As in equation (30), they are always linear in the bra-state correlation coefficients because of the nature of the NCCM approach. These equations were solved numerically via a standard linear decomposition package once the ket-state correlation coefficients had been determined.

We now use the ket and the bra states to define a measure of the quantum fluctuations in these systems. Firstly, we should note that the expectation value of the unrotated $s^{z}$ operator is zero for all values of $\Delta$ as our model state lies completely in the $x y$ plane. Hence we construct a measure of the quantum fluctuations by evaluating the sublattice magnetization in terms of the unrotated $s^{x}$ operator. After the local axes of the spins have been rotated this is described by

$$
M=\frac{1}{N} \sum_{i=1}^{N}\left|\left\langle\tilde{\Psi}\left|\sigma_{i}^{z}\right| \Psi\right\rangle\right|
$$

which is easily evaluated at the LSUB2 level of approximation, giving

$$
M=1-4 \tilde{b}_{1} b_{1} .
$$

This quantity is easily determined once the bra- and ket-state equations have been solved and is shown in figures 4 and 5. Results for higher-order LSUB $n$ approximations have been calculated, and are also shown in figures 4 and 5, and in table 4 .

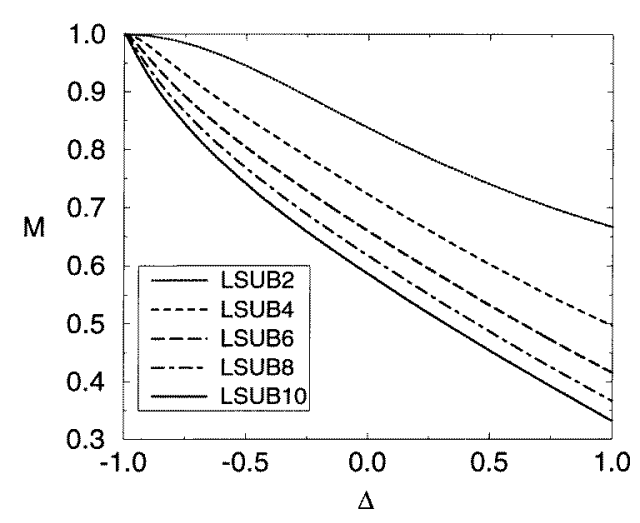

Figure 4. Results for the 1D CCM sublattice magnetization.

In $2 \mathrm{D}$, the sublattice magnetization in the region $-1<\Delta<1$ appears to converge to a non-zero value as one increases the approximation level. Our results therefore indicate non-zero, in-plane long-range order in the region $-1<\Delta<1$. In 1D, our results for the sublattice magnetization show a monotonic decrease with increasing LSUB $n$ for all values of $\Delta$. However, it is difficult at these levels of approximation to predict whether our results would eventually converge to zero. 


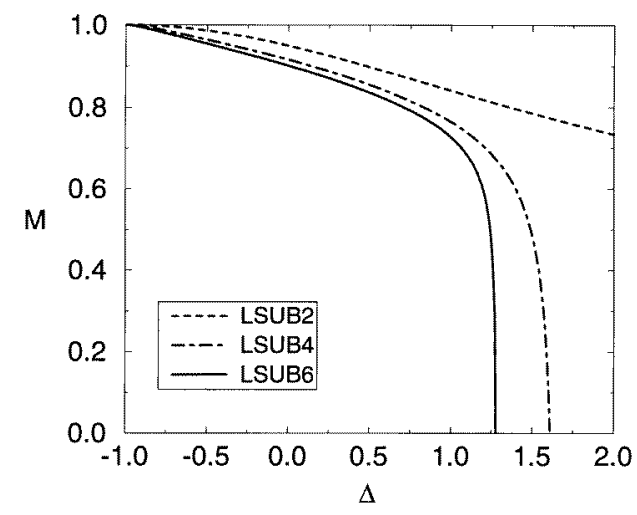

Figure 5. Results for the 2D CCM sublattice magnetization.

Table 4. Results for the 2D CCM sublattice magnetization for the calculations based on the planar model state.

\begin{tabular}{rlll}
\hline \multicolumn{1}{l}{$\Delta$} & LSUB2 & LSUB4 & LSUB6 \\
\hline-1.0 & 1.0 & 1.0 & 1.0 \\
-0.5 & 0.9865 & 0.9641 & 0.9545 \\
0.0 & 0.9496 & 0.9158 & 0.9014 \\
0.5 & 0.8983 & 0.8554 & 0.8366 \\
1.0 & 0.8414 & 0.7648 & 0.7273 \\
\hline
\end{tabular}

\section{Conclusion}

In this paper we have extended the CCM treatment of the $X X Z$ model for the 1D chain and the 2D square lattice to include the region $-1<\Delta<1$. We have obtained ground-state energies over the region $-1<\Delta<1$ which compare very favourably to exactly known results in 1D and Monte Carlo results in 2D. For the 2D square lattice our results indicate that there is non-zero Néel order in the $x y$ plane over the whole of this region. The 1D results for the sublattice magnetization were found to be inconclusive at these levels of approximation.

The ferromagnetic phase transition points were detected using both the LSUB $n$ and SUB2 schemes in 1D and 2D. The SUB2 scheme detected the ferromagnetic phase transition points precisely, which is very encouraging as the CCM is an ab initio calculation. The antiferromagnetic phase transition points were detected using the CCM, with SUB2 terminating points at $\Delta=1.7126$ in $1 \mathrm{D}$ and at $\Delta=1.204$ in 2D. In 2D, the LSUB4 and LSUB6 schemes also give critical points, corresponding to the antiferromagnetic transition, at $\Delta=1.648$ and $\Delta=1.286$ respectively.

It was also shown that the SUB2 calculation gave a critical exponent of $\alpha=1.50 \pm 0.01$ for the $1 \mathrm{D}$ ground-state energy near the ferromagnetic phase transition point. This result was found to be in good agreement to that of the exact ground-state energy. In contrast, all of the LSUB $n$ approximations yielded $\alpha=2.00 \pm 0.01$. We note, however, that, for the LSUB $n$ sequence of results in figure 3, the extracted values of the coefficient $A$ in equation (25) depend strongly on the truncation index $n$. We can readily refine our critical analysis in this case by performing a variant of the coherent anomaly method of Suzuki 
[26]. We have described such an analysis elsewhere [27] in the context of a similar CCM analysis [12] of the phase transition point at or near $\Delta=1$ for the same $X X Z$ model on the $2 \mathrm{D}$ square lattice as studied in the present paper. In that earlier work, however, we employed the Néel state with spins constrained to lie along the $z$-axis as the CCM model state, rather than the similar planar model state employed here with spins constrained to lie along the $x$-axis. Such an analysis of the coherent anomaly contained in the coefficient $A$ has been shown to lead to a powerful and consistent CCM analysis of the critical behaviour, and we do not repeat a similar discussion here, except to report that the SUB2 and LSUBn values for the critical exponent are thereby brought into agreement.

We note that, although the Hamiltonian remains the same, the properties of the regime $-1<\Delta<1$ are very different to those in the Ising-like regime, for $\Delta>1$. For example, in 1D this planar regime is believed to be critical, and with no gap in the excitation spectrum. This difference in behaviour therefore provides the physical motivation for investigating this regime and extending our knowledge of the $X X Z$ model.

We have found that our model state provides a much better starting point for the CCM calculations in the region $-1<\Delta<1$ than a model state which is the classical Néel state with spins lying parallel to the $z$-axis. In principle, the CCM can provide an accurate representation of the ground state by employing any model state, assuming only that the model state is not orthogonal to the exact ground state. In practice, the level of accuracy depends on the appositeness of the model state, and so our results support the idea that the ground-state wavefunction in the region $-1<\Delta<1$ has spins lying in the $x y$ plane.

\section{Acknowledgments}

We would like to thank Chen Zeng, Neil Davidson and Niels Walet for their interesting and helpful discussions. Two of us (R F Bishop and J B Parkinson) gratefully acknowledge a research grant from the Engineering and Physical Sciences Research Council (EPSRC) of Great Britain.

\section{References}

[1] Coester F 1958 Nucl. Phys. 7421

Coester F and Kümmel H 1960 Nucl. Phys. 17477

[2] Čižek J 1966 J. Chem. Phys. 45 4256; 1969 Adv. Chem. Phys. 1435

[3] Paldus J, Čižek J and Shavitt I 1972 Phys. Rev. A 550

[4] Bishop R F and Lührmann K H 1978 Phys. Rev. B 173757

[5] Kümmel H, Lührmann K H and Zabolitzky J G 1978 Phys Rep. C 361

[6] Arponen J S 1983 Ann. Phys., NY 151311

[7] Bishop R F and Kümmel H 1987 Phys. Today 40(3) 52

[8] Arponen J S, Bishop R F and Pajanne E 1987 Phys. Rev. A 36 2519, 2539; 1987 Condensed Matter Theories ed P Vashishta, R K Kalia and R F Bishop (New York: Plenum) vol 2, p 357

[9] Bartlett R J 1989 J. Phys. Chem. 931697

[10] Roger M and Hetherington J H 1990 Phys. Rev. B 41200

[11] Bishop R F, Parkinson J B and Xian Y 1991 Phys. Rev. B 449425

[12] Bishop R F, Hale R G and Xian Y 1994 Phys. Rev. Lett. 733157

[13] Farnell D J J and Parkinson J B 1994 J. Phys.: Condens. Matter 65521

[14] Bursill R, Gehring G A, Farnell D J J, Parkinson J B, Xiang Tao and Zeng Chen 1995 J. Phys.: Condens. Matter 78605

[15] Bishop R F, Parkinson J B and Xian Y 1993 J. Phys.: Condens. Matter 59169

[16] Anderson P W 1952 Phys. Rev. 86694 Oguchi T 1960 Phys. Rev. 117117

[17] Singh R R P 1989 Phys. Rev. B 399769 
Singh R R P and Huse D A 1989 Phys. Rev. B 407247

Zheng Weihong, Oitmaa J and Hamer C J 1991 Phys. Rev. B 438321

[18] Kubo K and Kishi T 1988 Phys. Rev. Lett. 612585

[19] Runge K J 1992 Phys. Rev. B 4512292,7229

[20] Carlson J 1989 Phys. Rev. B 40846

Trivedi N and Ceperley D M 1990 Phys. Rev. B 414552

[21] Barnes T, Kotchan D and Swanson E S 1989 Phys. Rev. B 394357

[22] Bethe H A 1931 Z. Phys. 71205

[23] Hulthén L 1938 Ark. Mat. Astron. Fys. A 26 No 111

[24] Orbach R 1958 Phys. Rev. 112309

Yang C N and Yang C P 1966 Phys. Rev. 150 321, 327

[25] Hellmann H 1935 Acta Physicochim. USSR I(6) 694

Feynman R P 1939 Phys. Rev. 56340

[26] Suzuki M 1986 J. Phys. Soc. Japan 554205

[27] Bishop R F, Xian Y and Zeng C 1996 Coherent Approaches to Fluctuations ed M Suzuki and N Kawashima (Singapore: World Scientific) p 125 